\title{
A GENERALIZATION OF CONTINUED FRACTIONS ${ }^{1}$
}

\author{
B. H. BISSINGER
}

1. Introduction. ${ }^{2}$ The generalizations and analogues of regular continued fractions due to Pierce [8], Lehmer [5], and Leighton [6] concern the iteration of rational functions to obtain rational approximations to a real number. The present generalization proceeds from the fact that the continued fraction

$$
\frac{1}{a_{1}+\frac{1}{a_{2}+\cdots}}
$$

can be written in the form

$$
f\left(a_{1}+f\left(a_{2}+\cdots\right.\right.
$$

where $f(t)=1 / t$. This suggests the possibility of using functions other than $1 / t$ to obtain generalizations of (1.1). In $\$ 2$ a class $F$ of functions which includes $1 / t$ is defined and in $\$ 3$ meaning is given to (1.2) for each $f \in F$ and each sequence $a_{1}, a_{2}, a_{3}, \cdots$ of positive integers. An algorithm is given for obtaining for a fixed $f \in F$ an expression of the form (1.2) corresponding to each number $x$ in the interval $0<x<1$; this expression is then called the $f$-expansion of $x$. The analogue of the $n$th convergent of a simple continued fraction is defined, and its behavior with respect to $x$ is noted. In $\S 4$ the form (1.2) is called an $f$-expansion when $f \in F$ and $a_{1}, a_{2}, a_{3}, \ldots$ is a sequence of positive integers. The convergence and some idea of the rapidity of convergence of an $f$-expansion are established. The one-to-one correspondence between $f$-expansions and $f$-expansions of numbers $x, 0<x<1$, is given in $\S 5$ by Theorem 5. In $\S 6$ statistical independence of the $a_{i}$ of an $f$-expansion is defined in the customary way and a subclass $F_{p}$ of $F$ for which the $a_{i}$ are statistically independent is considered. Various sets of numbers $x$ whose $f$-expansions are restricted by conditions on the $a_{i}$ are considered and the linear Lebesgue measures of these sets are given. In $\S 7$, when $f \in F_{p}$, certain sets of numbers $x$ which have been studied for $f(t)=1 / t$ by Borel [2] and F. Bernstein [1] are shown to be of measure zero.

Presented to the Society, September 12, 1943; received by the editors May 15, 1944.

1 The author wishes to thank Professors Agnew and Kac for their help in the preparation of this paper.

2 Numbers in brackets refer to the bibliography. 
2. The class $F$. Let $F$ denote the class of real functions $f(t)$ defined for $t \geqq 1$ and having the following properties:

$$
\begin{array}{cc}
f(1)=1 ; & \\
f\left(t_{1}\right)>f\left(t_{2}\right)>0, & 1 \leqq t_{1}<t_{2} \\
\lim _{t \rightarrow \infty} f(t)=0 ; & \\
\left|f\left(t_{2}\right)-f\left(t_{1}\right)\right|<\left|t_{2}-t_{1}\right|, & 1 \leqq t_{1}<t_{2}
\end{array}
$$

there is a constant $\lambda$ such that $0<\lambda<1$ and

$$
\left|f\left(t_{2}\right)-f\left(t_{1}\right)\right|<\lambda^{2}\left|t_{2}-t_{1}\right|, \quad 1+f(2)<t_{1}<t_{2} .
$$

3. The $f$-expansions of numbers. Let $f(t) \in F$ and $x$ be a fixed number, $0<x<1$. Let $z_{0}$ be defined by $x=f\left(z_{0}\right)$ and let the sequences $z_{1}, z_{2}, \cdots, \theta_{1}, \theta_{2}, \cdots$, and $a_{1}, a_{2}, \cdots$ be defined by the relations

$$
a_{n}=\left[z_{n-1}\right], \quad \theta_{n}=z_{n-1}-a_{n}, \quad \theta_{n}=f\left(z_{n}\right),
$$

for $n=1,2, \cdots$. If $\theta_{n} \neq 0$ for $n<k$ while $\theta_{k}=0$, we shall say the expansion terminates and that the $f$-expansion of $x$ is ${ }^{8}$

$$
f\left(a_{1}+f\left(a_{2}+\cdots+f\left(a_{k}\right) .\right.\right.
$$

In this case it is easy to see that $a_{k} \geqq 2$ and that the $f$-expansion of $x$ is equal to $x$. If $\theta_{n} \neq 0$ for $n=1,2, \cdots$, then the expansion will not terminate and we shall call

$$
f\left(a_{1}+f\left(a_{2}+\cdots\right.\right.
$$

the $f$-expansion of $x$.

By analogy with simple continued fractions we define

$$
x_{n}=f\left(a_{1}+f\left(a_{2}+\cdots+f\left(a_{n}\right)\right.\right.
$$

and call the elements of the sequence $x_{1}, x_{2}, \ldots$ the convergents of $x$. The integers $a_{1}, a_{2}, \cdots$ and the convergents $x_{1}, x_{2}, \cdots$ are uniquely determined by $x$ for almost all $x, 0<x<1$. When we wish to emphasize this functional dependence we shall write them in the form $a_{1}(x), a_{2}(x), \cdots$ and $x_{1}(x), x_{2}(x), \cdots$

To facilitate notation we introduce the function $\phi_{n}(t)$ defined when $f \in F$ and $a_{1}, a_{2}, \cdots$ is a sequence of positive integers by

$$
\phi_{n}(t)=f\left(a_{1}+f\left(a_{2}+\cdots+f\left(a_{n}+t\right), \quad t \geqq 0 .\right.\right.
$$

A simple induction proves the following lemma.

\footnotetext{
${ }^{3}$ In (3.2) and similar expressions we shall use a single parenthesis on the right.
} 
LEMMA 1. The function $\phi_{n}(t)$ is a decreasing (increasing) function of $t$ when $n$ is odd (even).

THEOREM 1. If $f \in F$ and $0<x<1$, then the odd (even) convergents of the f-expansion of $x$ form a decreasing (increasing) sequence bounded below (above) by $x$; thus

$$
0<x_{2}<x_{4}<\cdots \leqq x \leqq \cdots<x_{3}<x_{1} \leqq 1 .
$$

When $\phi_{n}(t)$ is defined by (3.5), we have $x_{n}=\phi_{n}(0), x=\phi_{n}\left(\theta_{n}\right)$, and $x_{n+1}=\phi_{n}\left(f\left(a_{n+1}\right)\right)$. Since $f\left(a_{n+1}\right) \geqq \theta_{n}>0$, we can apply Lemma 1 to obtain $x_{n}>x \geqq x_{n+1}$ when $n$ is odd and $x_{n}<x \leqq x_{n+1}$ when $n$ is even. Since $f\left(a_{n+1}+f\left(a_{n+2}\right)\right)>0$ and $x_{n+2}=\phi_{n}\left(f\left(a_{n+1}+f\left(a_{n+2}\right)\right)\right)$, we similarly have $x_{n}>x_{n+2}$ when $n$ is odd and $x_{n}<x_{n+2}$ when $n$ is even. These inequalities establish Theorem 1.

Corollary. If $\lim _{n \rightarrow \infty} x_{n}$ exists, then $\lim _{n \rightarrow \infty} x_{n}=x$.

4. Convergence of $f$-expansions. If $f \in F$ we shall mean by an $f$-expansion either a finite expansion $f\left(a_{1}+f\left(a_{2}+\cdots+f\left(a_{k}\right)\right.\right.$ in which the $a_{i}$ are positive integers and $a_{k} \geqq 2$, or an infinite expansion $f\left(a_{1}+f\left(a_{2}+\cdots\right.\right.$ in which the $a_{i}$ are positive integers. It is to be proved later that each $f$-expansion is generated by a unique $x$; meanwhile this is not assumed.

THEOREM 2. Let $f \in F$. If sequences $x_{n}$ and $y_{n}$ are defined in terms of an $f$-expansion by the formulas

$$
\begin{aligned}
& x_{n}=f\left(a_{1}+f\left(a_{2}+\cdots+f\left(a_{n}\right),\right.\right. \\
& y_{n}=f\left(a_{1}+f\left(a_{2}+\cdots+f\left(a_{n}+1\right),\right.\right.
\end{aligned}
$$

then

$$
0<x_{2}<x_{4}<\cdots<x_{3}<x_{1} \leqq 1
$$

and

$$
x_{n+1} \in I\left(a_{1}, a_{2}, \cdots, a_{n}\right),
$$

where $I\left(a_{1}, a_{2}, \cdots, a_{n}\right)$ is the closed interval with end points at $x_{n}$ and $y_{n}$.

Proof of (4.3) is identical with a part of the proof of (3.6). The conclusion (4.4) follows from Lemma 1 since $x_{n}=\phi_{n}(0)$,

$$
x_{n+1}=\phi_{n}\left(f\left(a_{n+1}\right)\right), \quad y_{n}=\phi_{n}(1), \quad \text { and } \quad 0<f\left(a_{n+1}\right) \leqq 1 .
$$

Lemma 2.4 Let $f \in F$. For a fixed positive integer $n$, the least upper

- We use the symbol $|E|$ to denote the linear Lebesgue measure of a set $E$. 
bound of $\left|I\left(a_{1}, a_{2}, \cdots, a_{n}\right)\right|$ for all sequences of positive integers $a_{i}$ is less than $\lambda^{n-2}$ where $\lambda$ is the constant in (2.5); that is, if $f \in F$ and

(4.5) $A_{n}=\underset{a_{1}, \cdots, a_{n} \geqq 1}{\text { l.u.b. }} \mid f\left(a_{1}+\cdots+f\left(a_{n}+1\right)-f\left(a_{1}+\cdots+f\left(a_{n}\right) \mid\right.\right.$,

where $a_{1}, a_{2}, \cdots, a_{n}$ assume independently all positive integral values, then

$$
A_{n} \leqq \lambda^{n-2}, \quad n=1,2, \cdots
$$

For $n \geqq 1$, we can write

$$
\begin{aligned}
A_{n+2} & =\underset{a_{1}, \cdots, a_{n+2} \geqq 1}{\text { l.u.b. }} \frac{\left|I\left(a_{1}, \cdots, a_{n+2}\right)\right|}{\left|I\left(a_{3}, \cdots, a_{n+2}\right)\right|} \cdot\left|I\left(a_{3}, \cdots, a_{n+2}\right)\right| \\
& \leqq A_{n} \cdot \underset{\substack{\text { l.u.b. } \\
a_{1}, a_{2} \geqq 1 ; 0<u<v \leqq 1}}{\left|\frac{f\left(a_{1}+f\left(a_{2}+u\right)-f\left(a_{1}+f\left(a_{2}+v\right)\right.\right.}{u-v}\right|}
\end{aligned}
$$

from which we obtain

$$
\begin{aligned}
A_{n+2} \leqq A_{n} \cdot{\underset{a}{a_{1}, a_{2} \geqq 1 ; 0<u<v \leqq 1}}_{\text {l.u.b. }}\left|\frac{f\left(a_{1}+f\left(a_{2}+u\right)-f\left(a_{1}+f\left(a_{2}+v\right)\right.\right.}{\left[a_{1}+f\left(a_{2}+u\right)\right]-\left[a_{1}+f\left(a_{2}+v\right)\right]}\right| \\
\cdot\left|\frac{f\left(a_{2}+u\right)-f\left(a_{2}+v\right)}{u-v}\right| .
\end{aligned}
$$

If $a_{2}=1$, then $a_{1}+f\left(a_{2}+u\right)>a_{1}+f\left(a_{2}+v\right) \geqq 1+f(2)$ when $a_{1}$ is a positive integer and $0<u<v \leqq 1$, so that by (2.5) and (2.4) the first and second factors of the product of which the least upper bound is taken in (4.7) are less than $\lambda^{2}$ and 1 , respectively. If $a_{2} \geqq 2>1+f(2)$, then the first and second factors are less than 1 and $\lambda^{2}$, respectively. So we have $A_{n+2} \leqq \lambda^{2} A_{n}, n=1,2, \cdots$. Since $A_{2} \leqq A_{1}<1$, the statement (4.6) follows easily by mathematical induction.

THEOREM 3. If $f \in F$, then each infinite f-expansion converges to $a$ number $x$ in the interval $0<x<1$; moreover

$$
\left|x_{n}-x\right| \leqq \lambda^{n-2}, \quad n=1,2, \cdots,
$$

where $\lambda$ is the constant in (2.5).

From Theorem 2 and Lemma 2 we conclude that $\left|x_{n+1}-x_{n}\right| \leqq \lambda^{n-2}$ for $n=1,2, \cdots$ and since $0<\lambda<1, x_{n}$ converges to a number $x$ which by (4.3) lies in each of the intervals from $x_{n}$ to $x_{n+1}$. This proves (4.8).

ThEOREM 4. If $f \in F$ and $0<x<1$, the f-expansion of $x$ converges to $x$. In the terminating case the $f$-expansion of $x$ obviously equals $x$ 
and in this sense converges to $x$. In the non-terminating case the conclusion follows directly from Theorem 3 and the corollary to Theorem 1.

Henceforth we shall use the notation $x=f\left(a_{1}+f\left(a_{2}+\cdots\right.\right.$ to mean that the $f$-expansion on the right side converges to $x$.

When $f(t)=1 / t$, the least upper bound of $|f(x)-f(y)| /|x-y|$ for $3 / 2<x<y$ is $(2 / 3)^{2}$, and so we may take $\lambda=2 / 3$. It follows from (4.8) that

$$
\left|x_{n}(x)-x\right| \leqq(2 / 3)^{n-2}, \quad n=1,2, \cdots .
$$

From the theory of simple continued fractions we know $[7,4]$ that

$$
\left|x_{n}(x)-x\right| \leqq z^{n-1}, \quad n=1,2, \cdots,
$$

where $z=\left(3-5^{1 / 2}\right) / 2$. Comparison of (4.9) and (4.10) shows that our method of obtaining estimates of the rapidity of uniform convergence of $f$-expansions gives, when applied to $f(t)=1 / t$, an estimate which is similar in form to the stronger estimate of (4.10).

5. Uniqueness. In this section we establish a one-to-one correspondence between $f$-expansions and $f$-expansions of numbers $x$, $0<x<1$. We note, as in simple continued fractions [7, p. 22], the following lemma.

LEMma 3. If $f \in F$, then any two of the three equations

$$
\begin{aligned}
& x=f\left(a_{1}+f\left(a_{2}+\cdots,\right.\right. \\
& y=f\left(a_{n}+f\left(a_{n+1}+\cdots,\right.\right. \\
& x=f\left(a_{1}+f\left(a_{2}+\cdots+f\left(a_{n-1}+y\right)\right.\right.
\end{aligned}
$$

implies the third, the f-expansions in (5.1) and (5.2) being infinite.

The proof of Lemma 3 is straightforward.

THEOREM 5. If $f \in F$ and $0<x<1$, then an $f$-expansion which converges to $x$ and the f-expansion of $x$ are identical.

If the two infinite $f$-expansions $f\left(a_{1}+f\left(a_{2}+\cdots\right.\right.$ and $f\left(b_{1}+f\left(b_{2}+\cdots\right.\right.$ converge to the same $x$, then by successively applying Lemma 3 we obtain $a_{n}=b_{n}, n=1,2, \ldots$. A similar argument proves that an infinite $f$-expansion and a finite $f$-expansion or two different finite $f$-expansions do not converge to the same $x$. Theorem 4 completes the proof.

6. Statistical independence. From (3.6) and (4.4) we see that $I\left(c_{1}, c_{2}, \cdots, c_{i}\right)$ except for at most its end points is identical with the 
set of $x, 0<x<1$, for which $a_{j}(x)=c_{j}, j=1,2, \cdots, i$. More exactly we have ${ }^{5}$

$$
\begin{aligned}
E\left[a_{j}(x)\right. & \left.=c_{j} ; j=1,2, \cdots, i\right] \\
& =I\left(c_{1}, c_{2}, \cdots, c_{i}\right)-\left\{f \left(c_{1}+f\left(c_{2}+\cdots+f\left(c_{i}+1\right)\right\}\right.\right.
\end{aligned}
$$

unless $i=1$ and $c_{1}=1$ in which case

$$
E\left[a_{1}(x)=1\right]=I(1)-\{f(1)\}-\{f(2)\} .
$$

LEMMA 4. If $f \in F$ and $c_{1}, c_{2}, \cdots, c_{n}$ and $c_{1}^{\prime}, c_{2}^{\prime}, \cdots, c_{n}^{\prime}$ are two sets of positive integers such that for at least one $j, 1 \leqq j \leqq n, c_{j} \neq c_{j}^{\prime}$, then the intervals $I\left(c_{1}, c_{2}, \cdots, c_{n}\right)$ and $I\left(c_{1}^{\prime}, c_{2}^{\prime}, \cdots, c_{n}^{\prime}\right)$ have at most an end point in common.

The proof of this lemma follows from (6.1) and (6.2) and from the fact that the sets $E\left[a_{j}(x)=c_{j} ; j=1,2, \cdots, n\right]$ and $E\left[a_{j}(x)=c_{j}^{\prime}\right.$; $j=1,2, \cdots, n]$ are mutually exclusive by Theorem 5 .

\section{Corollary. If $f \in F$, then}

$$
I\left(c_{1}, c_{2}, \cdots, c_{n}\right)=\left\{f\left(c_{1}+\cdots+f\left(c_{n}\right)\right\}+\sum_{j=1}^{\infty} I\left(c_{1}, c_{2}, \cdots, c_{n}, j\right) .\right.
$$

If $y_{1}, y_{2}, \cdots$ is a decreasing sequence of positive numbers such that $y_{1}=1$ and $y_{n} \rightarrow 0$ and $f(t)$ is the function whose graph is the polygon joining in order the points $\left(n, y_{n}\right), n=1,2, \cdots$, then $f(t) \in F$. Let $F_{p}$ be the class of all such polygonal functions.

THEOREM 6. If $f \in F_{p}$, then for any positive integers $i$ and $k$

$$
\left|E\left[a_{i}(x)=k\right]\right|=f(k)-f(k+1) .
$$

By (6.1) and (6.2) we have $\left|E\left[a_{1}(x)=k\right]\right|=|I(k)|=f(k)-f(k+1)$. For any positive integer $m$, it follows from (6.1) and Lemma 4 that $\left|E\left[a_{m+1}(x)=k\right]\right|=\sum\left|I\left(b_{1}, b_{2}, \cdots, b_{m}, k\right)\right|$ where $\sum$ is to be taken independently over all positive integral values of $b_{1}, b_{2}, \cdots, b_{m}$. By the mean value theorem we have

$$
\begin{aligned}
& \left|E\left[a_{m+1}(x)=k\right]\right| \\
& =\sum \mid f\left(b_{1}+\cdots+f(k+1)-f\left(b_{1}+\cdots+f(k) \mid\right.\right. \\
& =\sum\left|f\left(b_{1}\right)-f\left(b_{1}+1\right)\right| \mid f\left(b_{2}+\cdots+f(k+1)-f\left(b_{2}+\cdots+f(k) \mid\right.\right. \\
& =\left(\sum\left|f\left(b_{1}\right)-f\left(b_{1}+1\right)\right|\right) \cdot\left(\sum\left|I\left(b_{2}, \cdots, b_{m}, k\right)\right|\right) \\
& =\sum\left|I\left(b_{2}, \cdots, b_{m}, k\right)\right|=\left|E\left[a_{m}(x)=k\right]\right| .
\end{aligned}
$$

s The symbol $E[\ldots]$ shall denote the set of $x$ satisfying the proposition in brackets. 
An induction completes the proof.

The functions $a_{i}(x), i=1,2, \cdots$, are said to be statistically independent [4] if for each set of positive integers $n_{1}<n_{2}<\cdots<n_{m}$ and each set of positive integers $c_{1}, c_{2}, \cdots, c_{m}$

$$
\left|E\left[a_{n_{j}}(x)=c_{j} ; j=1,2, \cdots, m\right]\right|=\prod_{j=1}^{m}\left|E\left[a_{n_{j}}(x)=c_{i}\right]\right| .
$$

THEOREM 7. If $f \in F_{p}$, then the functions $a_{i}(x), i=1,2, \cdots$, are statistically independent.

The equation (6.3) is trivial for $m=1$. By (6.1) and Lemma 4 we have

$$
\begin{aligned}
\mid E\left[a_{n_{j}}(x)=c_{j} ; j=\right. & 1,2, \cdots, m] \mid \\
& =\sum^{\prime}\left|I\left(b_{1}, \cdots, b_{n 1-1}, c_{1} b_{n 1+1}, \cdots, c_{2}, \cdots, c_{m}\right)\right|
\end{aligned}
$$

where $\sum^{\prime}$ is to be taken independently over all positive integral values of $b_{i}$ for all indices $i$ from one to $n_{m}$ excepting $i=n_{1}, n_{2}, \cdots, n_{m}$. By an argument similar to that used in the proof of Theorem 6 we obtain

$$
\begin{aligned}
\mid E\left[a_{n_{j}}(x)\right. & \left.=c_{j} ; j=1,2, \cdots, m\right] \mid \\
& =\sum^{\prime}\left|f\left(b_{1}\right)-f\left(b_{1}+1\right)\right| \cdot\left|I\left(b_{2}, \cdots, b_{n_{1}-1}, c_{1}, \cdots, c_{m}\right)\right| \\
& =\sum^{\prime}\left|I\left(b_{2}, \cdots, b_{n_{1}-1}, c_{1}, \cdots, c_{m}\right)\right|=\cdots \\
& =\sum^{\prime}\left|I\left(c_{1}, b_{n_{1}+1}, \cdots, c_{m}\right)\right| \\
& =\left|f\left(c_{1}\right)-f\left(c_{1}+1\right)\right| \cdot\left(\sum^{\prime}\left|I\left(b_{n_{1}+1}, \cdots, c_{m}\right)\right|\right) \\
& =\left|E\left[a_{n_{1}}(x)=c_{1}\right]\right| \cdot\left|E\left[a_{n_{j}}(x)=c_{j} ; j=2, \cdots, m\right]\right|
\end{aligned}
$$

and again an induction completes the proof.

CoRollary. If $f \in F_{p}$, then for each set of positive integers $n_{1}<n_{2}<\cdots<n_{m}$ and each set of positive integers $c_{1}, c_{2}, \cdots, c_{m}$, $d_{1}, d_{2}, \cdots, d_{m}$ such that $c_{j} \leqq d_{j}, j=1,2, \cdots, m$, we have

$$
\begin{aligned}
\left|E\left[c_{j} \leqq a_{n_{j}}(x) \leqq d_{j} ; j=1,2, \cdots, m\right]\right| & =\prod_{j=1}^{m}\left|E\left[c_{j} \leqq a_{n_{j}}(x) \leqq d_{i}\right]\right| \\
& =\prod_{j=1}^{m}\left|f\left(c_{j}\right)-f\left(d_{j}+1\right)\right| .
\end{aligned}
$$

7. Sets of measure zero. ${ }^{\circ}$ The results of $\$ 6$ will now be used in order to prove a few measuretheoretical facts concerning $f$-expansions under the assumption that $f \in F_{p}$.

- Theorems, similar to those in this section, applying to the simple continued fraction have been proved by Borel [2] and Bernstein [1]; for expositions see [3]. 
TheOREM 8. If $f \in F_{p}$, then the set of $x, 0<x<1$, for which the sequence $a_{1}(x), a_{2}(x), \cdots$ is bounded, has measure zero.

Let the set $E\left[a_{i}(x) \leqq k ; i=1,2, \cdots, m\right]$ be denoted by $G_{k}^{m}$. In the corollary to Theorem 7 we set $n_{j}=j, c_{j}=1, d_{j}=k$ and obtain

$$
\left|G_{k}^{m}\right|=\prod_{j=1}^{m}\{1-f(k+1)\}=\{1-f(k+1)\}^{m} .
$$

If we let $G_{k}=E\left[a_{i}(x) \leqq k ; i=1,2, \cdots\right]$, then $G_{k} \in G_{k}^{m}, m=1,2, \cdots$, and so $\left|G_{k}\right|=0$. The set of $x, 0<x<1$, for which the sequence $a_{1}(x), a_{2}(x), \cdots$ is bounded is $G=\sum_{i=1}^{\infty} G_{i}$ and consequently $|G|=0$.

Similarly the set of $x, 0<x<1$, for which $a_{i}(x)>k, i=1,2, \cdots, m$, has measure $\{f(k+1)\}^{m}$. An argument similar to that used in the proof of Theorem 8 proves the following theorem.

THEOREM 9. If $f \in F_{p}$, then the set of $x, 0<x<1$, for which $a_{i}(x)>1$, $i=1,2, \cdots$, has measure zero.

THEOREM 10. If $f \in F_{p}$ and $\phi(1), \phi(2), \ldots$ is a sequence of positive integers for which

$$
\sum_{n=1}^{\infty} f(\phi(n)+1)
$$

is divergent, then the set of $x, 0<x<1$, for which $a_{n}(x) \leqq \phi(n)$, $n=1,2, \cdots$, has measure zero.

Let $H_{m}=E\left[a_{i}(x) \leqq \phi(i) ; i=1,2, \cdots, m\right]$. By an argument similar to the one used in proving Theorem 8 we have

$$
\left|H_{m}\right|=\prod_{i=1}^{m}\{1-f(\phi(i)+1)\} .
$$

Since $0<f(\phi(i)+1)<1$ for $i=1,2, \cdots$, the divergence of the series (7.1) is equivalent to the limit as $m \rightarrow \infty$ of the product in (7.2) being zero. If we let $H=E\left[a_{i}(x) \leqq \phi(i) ; i=1,2, \ldots\right]$, then since $H \in H_{m}$ for every positive integer $m$, it follows that $|H|=0$.

The last three theorems can be generalized to infinite subsequences of the sequence $a_{1}(x), a_{2}(x), \cdots$.

\section{BIBLIOGRAPHY}

1. F. Bernstein, Über eine Anwendung der Mengenlehre auf ein aus der Theorie der säkularen Störungen herrührendes Problem, Math. Ann. vol. 71 (1912) pp. 417-439, especially $\$ 2$. 
2. E. Borel, Les probabilités dénombrables et leurs applications arithmétiques, Rend. Circ. Mat. Palermo vol. 27 (1909) pp. 246-271.

3. G. H. Hardy and E. M. Wright, Introduction to the theory of numbers, Oxford, 1938, chaps. 10-11.

4. M. Kac, Sur les fonctions independantes, I, Studia Math. vol. 6 (1936) pp. 46-58.

5. D. H. Lehmer, $A$ cotangent analogue of continued fractions, Duke Math. J. vol. 4 (1938) pp. 323-340.

6. W. Leighton, Proper continued fractions, Amer. Math. Monthly vol. 47 (1940) pp. 274-280.

7. O. Perron, Die Lehre von den Kettenbriichen, 2nd ed., Leipzig, 1929.

8. T. A. Pierce, On an algorithm and its use in approximating roots of an algebraic equation, Amer. Math. Monthly vol. 36 (1929) pp. 523-525.

CoRnell University 